\title{
Caracterización de lesiones duodenales y su sobrevida
}

\author{
Julio Núñez V. ${ }^{1,2,3}$, Sergio Rubel C. ${ }^{1}$, Gabriel Cavada C., ${ }^{3,4}$, Laura Segovia G. ${ }^{1}$, \\ Carlos Matus F. ${ }^{1}$, Antonio Sa Cunha ${ }^{5}$, Vilma Rodríguez A. ${ }^{1}$ y Rodrigo Abularach C. ${ }^{1}$
}

\section{Characterization of duodenal lesions and their survival}

Introduction: Duodenal lesions are infrequent. Aim: To characterize patients with duodenal lesions observed between January 2008 and December 2013 at the Southern Metropolitan Health Service, in Santiago, Chile. Materials and Method: Data were obtained from the Barros Luco Trudeau Hospital and El Pino Hospital records. The patients included were those observed between January 2008 and December 2013. Age, gender, clinical characteristics, location, diagnostic methods, anatomopathological findings and treatment were analyzed. On April $24^{\text {th }} 2019$ was obtained the date of death of all patients at the National Civil Registry. The statistical analysis was performed with the software STATA 15.1. Results: 157 patients with duodenal lesions were found, 65 presented duodenal adenocarcinoma and 71 patients had duodenal polyps, 25 of them were adenomas. Univariate analysis of survival showed that patients with polyps associated with carcinoma and duodenal adenocarcinomas had a lower survival rate $(p=0.013$, HR 6.584 y $\mathrm{p}<0.001$, HR 7.604, respectively). In patients with duodenal adenocarcinoma, those who underwent surgery with curative intent, and those who receive chemotherapy had a better overall survival than those who did not received therapy ( $\mathrm{p}<0.001$, HR 0.351 y $\mathrm{p}=0.001$, HR 0.276 , respectively). Discussion: We propose endoscopic study in at-risk populations and treatment with curative intent for patients with duodenal adenocarcinoma without distant metastases. The estimated incidence of duodenal adenocarcinoma in our population is 1.1 per 100,000 inhabitants and seem to be higher than other published.

Key words: duodenal neoplasms; adenoma; small bowel cancer.

\section{Resumen}

Introducción: Las lesiones duodenales son infrecuentes. Objetivo: Caracterizar a los pacientes con lesiones duodenales observados entre enero de 2008 y diciembre de 2013 en el Servicio de Salud Metropolitano Sur, en Santiago de Chile. Materiales y Método: Se obtuvieron los datos de los registros del Hospital Barros Luco Trudeau y Hospital El Pino. Los pacientes incluidos fueron los observados entre enero de 2008 y diciembre de 2013. Se analizaron edad, género, características clínicas, localización, métodos diagnósticos, hallazgos anatomopatológicos y tratamiento. El 24 de abril de 2019 se obtuvo la fecha de fallecimiento de todos los pacientes del Registro Civil. El análisis estadístico se realizó con el software STATA 15.1. Resultados: Se encontraron 157 pacientes con lesiones duodenales, 65 presentaron adenocarcinoma duodenal y 71 pacientes pólipos duodenales, 25 de ellos fueron adenomas. Análisis univariado de sobrevida evidenció que los pacientes con pólipos asociado a carcinoma y los adenocarcinomas duodenales, presentaron una sobrevida inferior $(p=0,013$, HR 6,584 y p $<0,001$, HR 7,604, respectivamente). En los pacientes con adenocarcinoma duodenal, aquellos que se sometieron a una cirugía con intención curativa, y aquellos que recibieron quimioterapia tuvieron una sobrevida global mejor que aquellos que no recibieron terapia ( $\mathrm{p}<0,001$, HR 0,351 y $\mathrm{p}=0,001$, HR 0,276 , respectivamente. Discusión: La incidencia estimada del adenocarcinoma duodenal en nuestra población es de 1,1 por cada 100.000 habitantes, la que es más alta que otras publicadas. Proponemos estudio endoscópico en poblaciones de riesgo y tratamiento con intención curativa para los pacientes con adenocarcinoma duodenal sin metástasis a distancia.

Palabras clave: tumores duodenales; adenoma; cáncer intestino delgado.
Hospital Barros Luco Trudeau, Santiago de Chile. ${ }^{2}$ Hospital del Salvador, Santiago de Chile. ${ }^{3}$ Universidad de Chile, Santiago de Chile. ${ }^{4}$ Escuela de Salud Pública, Universidad de Chile, Santiago de Chile.

${ }^{5}$ Hôpital Paul Brousse, Villejuif. París, Francia.

Recibido el 26 de agosto de 2019 y aceptado para publicación el 13 de enero de 2020.

Correspondencia a:

Dr. Julio Núñez V.

Dr.julio.nunez@gmail.com 


\section{Introducción}

Dada su baja frecuencia, en la literatura existen pocos artículos que describan lesiones duodenales. Se presentan en trabajos que incluyen tumores de intestino delgado o periampulares. La baja incidencia contribuye a la baja sospecha clínica. La mayoría de ellas permanece en silencio durante largos períodos, o puede manifestarse con síntomas inespecíficos. Cuando los síntomas específicos aparecen habitualmente nos encontramos frente a una enfermedad avanzada ${ }^{1,2}$.

El objetivo general de este artículo es caracterizar los pacientes con lesiones duodenales observadas entre enero de 2008 y diciembre de 2013 en el Servicio Metropolitano de Salud del Sur (SSMS), en Santiago de Chile, y comparar los resultados con la literatura disponible.

\section{Materiales y Método}

Se realizó un estudio analítico y de cohorte histórica. Se analizaron lesiones neoplásicas del duodeno y de páncreas del SSMS en Santiago de Chile. El Servicio de Anatomía Patológica en el Complejo Asistencial Barros Luco Trudeau (CABLT) recibió y analizó las biopsias de los 5 hospitales de adultos. El hospital principal para adultos es CABLT, que resuelve la patología más compleja en SSMS. El CABLT cuenta con el Centro de Diagnóstico y Endoscopia de Tratamiento (CEDIT), que resuelve la mayoría de los procedimientos endoscópicos adultos del SSMS. Los hospitales El Pino y San Luis de Buin son hospitales de adultos de complejidad menor. La población asignada al servicio fue de 1.060.587 habitantes (Ministerio de Salud de Chile, año 2010).

Los datos analizados se obtuvieron de las bases de datos del Servicio de Anatomía Patológica del CABLT y el CEDIT, y los registros médicos de CABLT y el Hospital El Pino. Todas las lesiones neoplásicas de duodeno y de páncreas se incluyeron para un análisis posterior. Los pacientes incluidos fueron los observados entre enero de 2008 y diciembre de 2013.

Para lograr el propósito de analizar solo las lesiones duodenales, todos los informes y placas de biopsias fueron revisados por un médico anatomopatólogo. Se clasificaron en duodenitis, pólipo hiperplásico, pólipo adenomatoso, adenocarcinoma duodenal y otros tumores de duodeno. A su vez los pólipos adenomatosos se clasificaron en bajo grado, alto grado de displasia, y asociado a carcinoma.
El 24 de abril de 2019 se obtuvo la fecha de fallecimiento de todos los pacientes del Registro Civil de Chile. Posteriormente, se realizó un análisis según edad al momento del diagnóstico, sexo, tipo de lesión, síntomas, duración de los síntomas, estudio diagnóstico, etapa al momento del diagnóstico, segmento afectado y tratamiento.

El análisis estadístico se realizó con el software STATA 15.1. La sobrevida fue estimada a través del método de Kaplan-Meier, y las curvas de sobrevida comparada por el modelo de riesgos proporcionales de Cox.

\section{Resultados}

Se observaron 157 pacientes con lesiones duodenales, 76 de ellos presentaron diagnóstico de neoplasia duodenal maligna, de los cuales 65 tuvieron adenocarcinoma duodenal, los otros 11 pacientes del grupo fueron asociados a otros tipos de neoplasia maligna. Además, se observaron 71 pacientes con pólipos duodenales, de los cuales 46 correspondieron a pacientes con la variedad hiperplásica y 25 con pólipos adenomatosos. Por último 10 pacientes presentaron duodenitis (Figura 1).

Para los pacientes con lesiones duodenales $(\mathrm{n}=157), 46,5 \%$ fueron mujeres y $53,5 \%$ hombres. Los grupos fueron homogéneos en cuanto a género. La edad promedio fue de 62,6 años (DS 15,2 años).

Los pacientes con diagnóstico de pólipos hiperplásicos $(n=46)$ presentaron una edad promedio de 57,8 años (DS 15,6 años); los pacientes con pólipos adenomatosos ( $\mathrm{n}=25)$ 62,4 años (DS 14,3 años) y los con adenocarcinoma duodenal $(n=65)$ una edad promedio de 67,7 años (DS 12,9 años).

Dentro del grupo de pacientes con pólipos adenomatosos, encontramos 14 pacientes con displasia de bajo grado (edad promedio 61,7 años, DS 14,7), 9 pacientes con displasia de alto grado (edad promedio 62,2 años, DS 15,7$)$ y 2 pacientes con pólipo asociado a adenocarcinoma (edad promedio 68 años).

La diferencia etaria entre los pacientes con adenocarcinoma duodenal y los con lesiones poliposas fue estadísticamente significativa $(p=0,0005)$. Análisis univariado de sobrevida evidenció que los pacientes con pólipos asociado a carcinoma y los adenocarcinomas duodenales, presentaron una sobrevida inferior $(\mathrm{p}=0,013$, HR 6,584 y $\mathrm{p}<0,001$, HR 7,604, respectivamente).

La población asignada al SSMS fue de 1.060.587 habitantes, lo que permite inferir una incidencia en este grupo de adenocarcinoma duodenal de 1,1 por 
100.000 habitantes. Los datos se obtuvieron de la publicación del Ministerio de Salud de Chile (año de publicación 2010) (Tablas 1 y 2).

El 65,9\% de los pacientes con lesiones duodenales describieron síntomas en el momento previo al diagnóstico.

Existió un mayor porcentaje de pacientes con síntomas en el grupo de adenocarcinoma $(83,1 \%)$ que en los pacientes con pólipos (49,3\%). El principal síntoma manifestado por pacientes con adenocarcinoma duodenal en el momento del diagnóstico fue ictericia $(55,4 \%)$ y en los pacientes con pólipos fue el dolor abdominal (29,6\%) (Tabla 2).

La presencia de síntomas al momento del diagnóstico fue asociado a una menor sobrevida $(\mathrm{p}=0,001, \mathrm{HR} 2,202)$. Específicamente ictericia, compromiso del estado general y síndrome de mal vaciamiento gástrico. El diagnóstico de colangitis fue también asociado a menor sobrevida $(p=0,049$, HR 2,172).

\section{Métodos de estudio}

Todos los pacientes con pólipos duodenales tuvieron una endoscopia digestiva alta como instrumento de diagnóstico. Para los pacientes con adenocarcinoma duodenal, el 95,4\% de ellos utilizó este procedimiento. El $43,1 \%$ de los pacientes con adenocarcinoma requirió una colangiopancreatografía retrógrada endoscópica (CPRE), y el 75\% de ellos terminó con una prótesis biliar. El 64,6\% de los pacientes con adenocarcinoma se realizó una tomografía axial computada (TC) (Tabla 2).

La mayoría de los registros de lesiones duodenales se localizaron en la segunda porción del duodeno $(66,2 \%, n=104)$. La primera porción duodenal $(26,1 \%, \mathrm{n}=41)$ fue el segundo segmento más comprometido. El 62,5\% de los pacientes con lesiones en el segmento dos, tuvo compromiso de papila. Específicamente, para pacientes con adenocarcinoma duodenal, el 89,2\% $(\mathrm{n}=58)$ tuvo compromiso de la segunda porción duodenal. $15,9 \%$ de las lesiones, todas poliposas, se ubicaron entre el segmento uno y dos, sin precisar más detalle.

\section{Histología y estadio clínico}

Sesenta y cinco pacientes presentaron adenocarcinoma duodenal, de ellos 22 pacientes fueron sometidos a una duodenopancreatectomía cefálica, por ende, sólo fue posible el análisis del estadio anatomopatológico de estos pacientes (TNMp). De este grupo se observaron 14 pacientes con compromiso del espesor completo de la pared duodenal $(\mathrm{T} 4 \mathrm{p}+)$

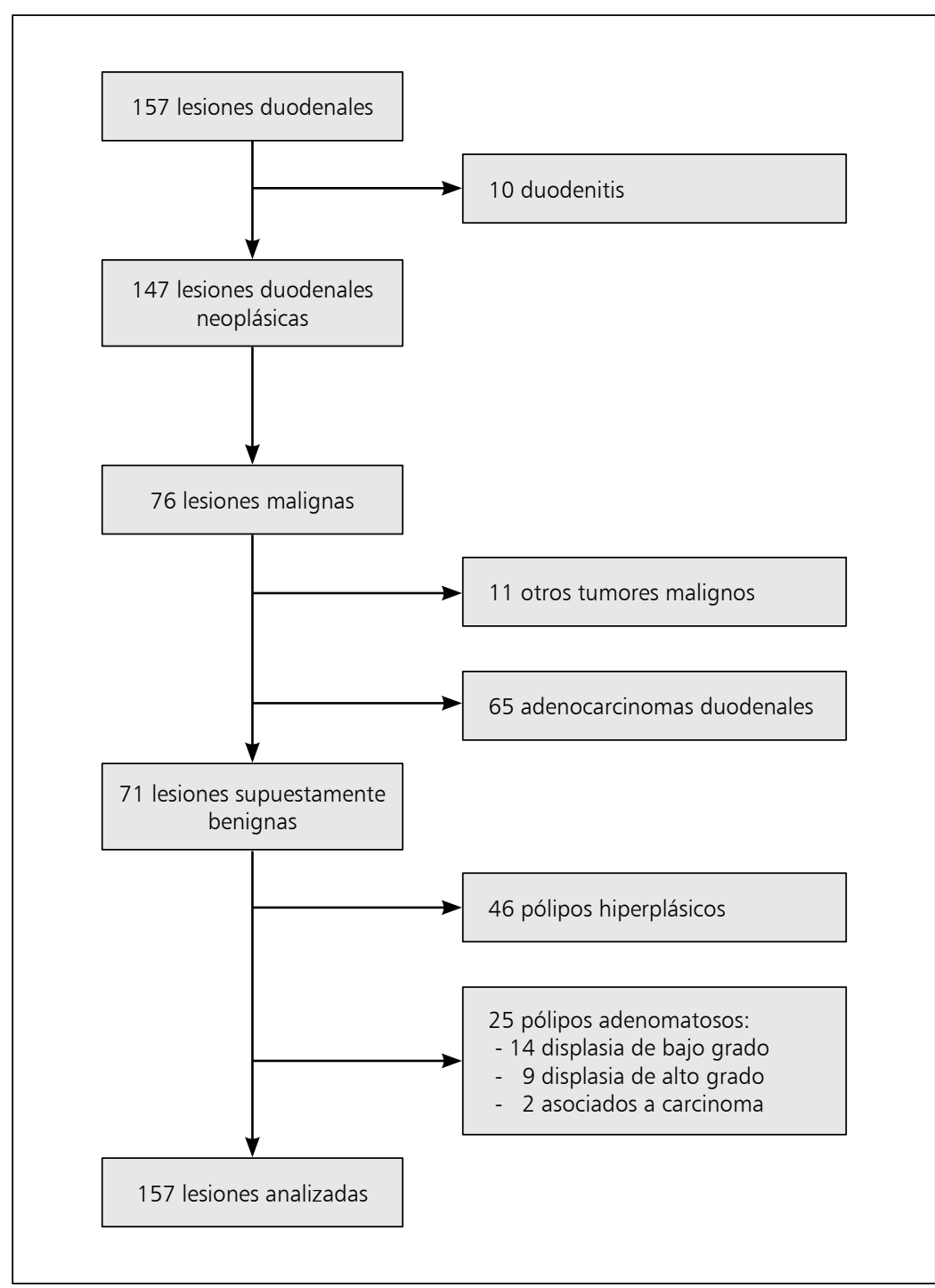

Figura 1.

Tabla 1

\begin{tabular}{|lccc|}
\hline & n & $\begin{array}{c}\text { Promedio de } \\
\text { edad (años) }\end{array}$ & $\begin{array}{c}\text { DS } \\
\text { (años) }\end{array}$ \\
1. Pólipos hiperplásicos & 46 & $57,8^{*}$ & 15,6 \\
2. Pólipos adenomatosos & 25 & $62,4^{\circ}$ & 14,3 \\
2.1. Displasia de bajo grado & 14 & 61,7 & 14,7 \\
2.2. Displasia de alto grado & 9 & 62,2 & 15,7 \\
2.3. Pólipos asociados a carcinoma & 2 & 68 & - \\
3. Adenocarcinomas duodenales & 65 & $67,7^{* \circ}$ & 12,8 \\
\hline
\end{tabular}

*Diferencia en edad significativa entre pólipo hiperplásico y adenocarcinoma duodenal. ${ }^{\circ}$ Diferencia en edad significativa entre pólipo adenomatoso y adenocarcinoma duodenal. 
Tabla 2. Caracterización de pacientes según tipo histológico de la pieza

\begin{tabular}{|c|c|c|c|}
\hline & Adenocarcinoma & Pólipo adenomatoso & Pólipo hiperplásico \\
\hline $\begin{array}{l}\text { Características generales } \\
\text { Número de pacientes } \\
\text { Edad promedio } \\
\text { Edad mediana } \\
\text { Edad DS } \\
\text { Sexo }(\mathrm{h} / \mathrm{m})\end{array}$ & $\begin{array}{l}65 \\
67,7 \text { años } \\
70 \text { años } \\
12,9 \text { años } \\
1,2\end{array}$ & $\begin{array}{l}25 \\
62,4 \text { años } \\
65 \text { años } \\
14,3 \text { años } \\
1,5\end{array}$ & $\begin{array}{l}46 \\
57,9 \text { años } \\
59,5 \text { años } \\
15,6 \text { años } \\
\quad 1\end{array}$ \\
\hline Síntomas previo al diagnóstico & $83,1 \%$ & $60 \%$ & $43,5 \%$ \\
\hline $\begin{array}{l}\text { Métodos de estudio } \\
\text { Endoscopía digestiva alta } \\
\text { TAC } \\
\text { CPRE } \\
\text { Prótesis biliar }\end{array}$ & $\begin{array}{l}95,4 \% \\
64,6 \% \\
43,1 \% \\
32,3 \%\end{array}$ & $\begin{array}{c}100 \% \\
36 \% \\
- \\
-\end{array}$ & $\begin{array}{l}100 \% \\
8,7 \% \\
- \\
-\end{array}$ \\
\hline $\begin{array}{l}\text { Localización } \\
\text { Compromiso Segmento } 2 \\
\text { Compromiso Segmento } 1 \\
\text { Compromiso de papila explicitado } \\
\text { Sin mención localización precisa }\end{array}$ & $\begin{array}{l}\mathrm{N}=58 \\
\mathrm{~N}=7 \\
\mathrm{~N}=45 \\
\mathrm{~N}=4\end{array}$ & $\begin{array}{l}\mathrm{N}=13 \\
\mathrm{~N}=9 \\
\mathrm{~N}=12 \\
\mathrm{~N}=4\end{array}$ & $\begin{array}{l}N=17 \\
N=19 \\
N=3 \\
N=15\end{array}$ \\
\hline $\begin{array}{l}\text { Histopatología } \\
\text { T4+P } \\
\text { N+P } \\
\text { M1 }\end{array}$ & $\begin{array}{l}\mathrm{N}=14 \\
\mathrm{~N}=11 \\
\mathrm{~N}=19\end{array}$ & $\begin{array}{c}- \\
- \\
N=1\end{array}$ & $\begin{array}{l}- \\
- \\
-\end{array}$ \\
\hline $\begin{array}{l}\text { Tratamiento } \\
\text { Polipectomía } \\
\text { Gastrectomía subtotal } \\
\text { DPC* } \\
\text { Quimioterapia } \\
\text { Radioterapia + quimioterapia }\end{array}$ & $\begin{array}{c}- \\
- \\
N=22 \\
N=10 \\
N=4\end{array}$ & $\begin{aligned} \mathrm{N} & =22 \\
\mathrm{~N} & =1 \\
\mathrm{~N} & =2 \\
& - \\
& -\end{aligned}$ & $\begin{aligned} N & =46 \\
& - \\
& - \\
& - \\
& -\end{aligned}$ \\
\hline
\end{tabular}

*Duodenopancreatectomía cefálica.

y 11 pacientes con compromiso ganglionar $(\mathrm{Np}+)$, ambos demostrados en la pieza quirúrgica.

Análisis univariado de sobrevida evidenció una sobrevida inferior para los pacientes con compromiso completo de la pared duodenal (T4+) $(\mathrm{p}=0,038, \mathrm{HR} 1,793)$, ganglios comprometidos $(\mathrm{N}+)(\mathrm{p}=0,002, \mathrm{HR} 2,27)$ y metástasis a distancia $(\mathrm{M} 1+)(\mathrm{p}<0,001, \mathrm{HR} 2,512)$, con respecto a otras lesiones duodenales.

Debido a la dificultad para diferenciar el adenocarcinoma duodenal del adenocarcinoma ampular, desde el punto de vista anatómico, ambos se colocaron en el mismo grupo de pacientes con adenocarcinoma duodenal con compromiso del segmento 2 $(\mathrm{n}=58)$ para su análisis (Tabla 2).

\section{Resultados y supervivencia a los 5 años}

En cuanto al procedimiento, la mayoría de los pacientes con pólipos se resolvieron mediante una polipectomía endoscópica $(n=68)$, el resto $(n=3)$ fueron sometidos a un procedimiento quirúrgico por alta sospecha de malignidad. De los 3 pacientes con pólipos sometidos a cirugía mayor, 2 eran pólipos asociados a carcinoma infiltrante, ambos al año de su diagnóstico se encontraban fallecidos. El otro paciente fue un pólipo de alto grado de displasia (Tabla 2 y Figura 2).

A 22 pacientes con adenocarcinoma duodenal se les realizó una duodeno-pancreatectomía cefálica (DPC), tal como se describió previamente. Este grupo de pacientes presentó una sobrevida global significativamente mejor que los no sometidos a este procedimiento ( $\mathrm{p}<0,001$, HR 0,351) (Tabla 2 y Figura 3).

De los pacientes con adenocarcinoma, 10 recibieron quimioterapia, la mayoría de ellos basados en el esquema de 5-fluorouracilo. Nueve de ellos fueron sometidos previamente a DPC. Cuatro pacientes de este grupo recibieron quimioterapia asociado a radioterapia. 
Aquellos pacientes que recibieron quimioterapia, presentaron una sobrevida global mayor que los pacientes pertenecientes al grupo de adenocarcinomas duodenales que no recibieron este tratamiento $(\mathrm{p}=0,001, \mathrm{HR} 0,276)$. Los que recibieron quimioterapia asociado a radioterapia, también presentaron una sobrevida global mayor $(p=0,017$, HR 0,173$)$ (Tabla 2 y Figura 4).

\section{Discusión}

El intestino delgado representa alrededor del $75 \%$ de la longitud del tracto digestivo, el cual comprende el segmento duodenal. A pesar de lo anterior, los tumores en esta localización son poco frecuentes ${ }^{3}$. Muchas razones podrían explicar este hecho ${ }^{4}$.

Primero, la característica del quimo, que al ser líquido produce un tránsito rápido, permitiendo un tiempo de contacto corto de carcinógenos con los enterocitos $^{4-6}$. El contenido gástrico ácido, se vacía de forma intermitente al duodeno, donde se neutraliza con bicarbonato del páncreas, hígado y de la mucosa duodenal. Esto crea fluctuaciones de $\mathrm{pH}$ amplias y rápidas en el duodeno, así como un gradiente de $\mathrm{pH}$ que conduce a una baja acidez del contenido del lumen en el yeyuno proximal. El fenómeno permite la neutralización del quimo ácido que llega del estómago, con un $\mathrm{pH}$ entre 2 a 5 , a un pH entre 5 y 6 a nivel duodenal.

En segundo lugar, una relación única entre el huésped y microbiota intestinal. El número de bacterias aumenta desde el estómago $\left(10^{1}\right.$ a $10^{3}$ bacterias $\left./ \mathrm{g}\right)$ hasta el colon $\left(10^{11}\right.$ a $10^{12}$ bacterias $\left./ \mathrm{g}\right)$. El intestino delgado comprende principalmente bacterias Gram positivas y aeróbicas, y el intestino grueso contiene bacterias predominantemente Gram negativas y anaeróbicas ${ }^{8}$.

Una menor carga bacteriana, particularmente de anaerobios, da como resultado una menor producción de carcinógenos. Existen varios factores que evitan el crecimiento excesivo de bacterias en este segmento, dentro de los que incluyen la secreción de jugo gástrico y bilis, que tienen un efecto antibacteriano. La protección mecánica producida por el peristaltismo impide la adherencia de bacterias a la mucosa intestinal y la válvula ileocecal previene la translocación retrógrada ${ }^{4-6,9}$.

El sistema inmune intestinal normal incluye mecanismos celulares y humorales. Asociado a lo anterior, existe una producción de mucina por células epiteliales que inhiben las bacterias patógenas ${ }^{10}$.

Finalmente, la capacidad de la mucosa para metabolizar y/o desintoxicar ciertos componentes, y la rápida rotación de las células epiteliales que sobrepasa el crecimiento y desarrollo de células neoplásicas, confiere la protección de este órgano contra neoplasias ${ }^{1,4-10}$.

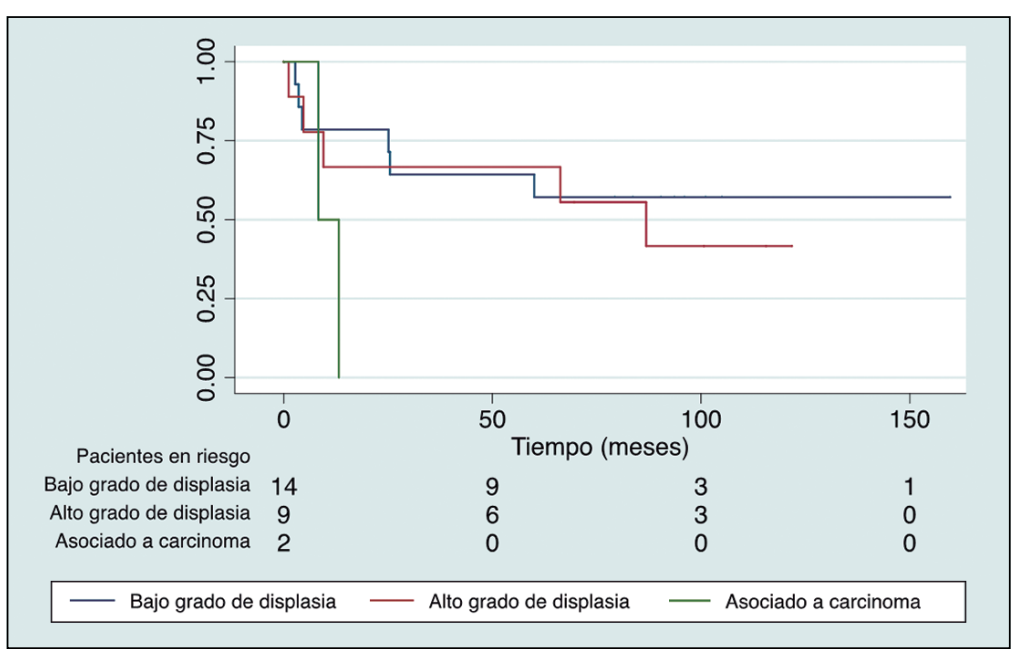

Figura 2. Sobrevida global en pacientes con pólipos adenomatosos. Mediana de seguimiento: 69,6 meses.



Figura 3. Sobrevida global de pacientes con adenocarcinoma duodenal sometidos o no a operación de Whipple. Mediana de seguimiento 5,3 meses.

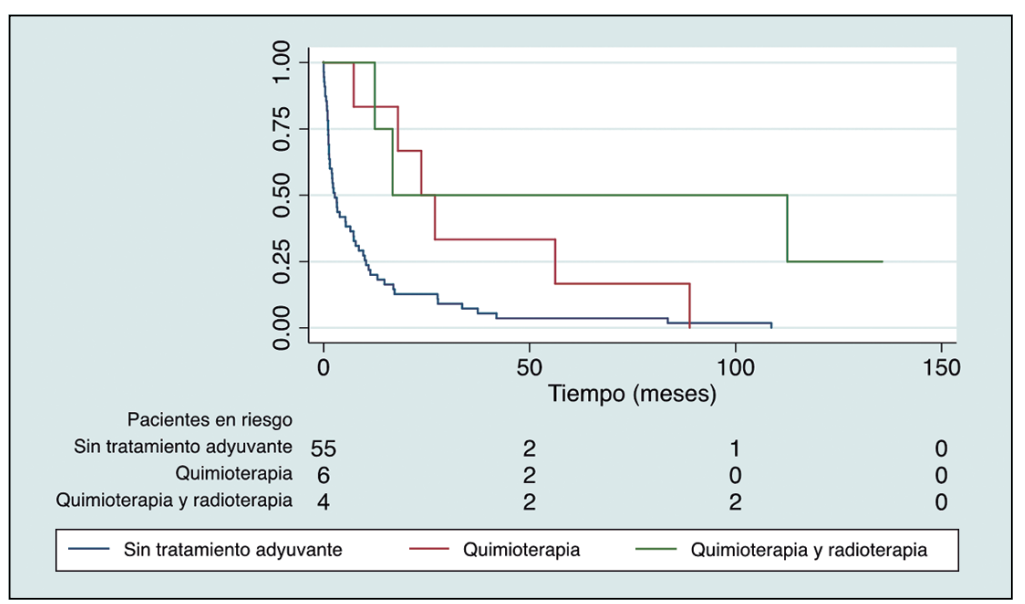

Figura 4. Sobrevida global de pacientes con adenocarcinoma duodenal según tratamiento adyuvante. Mediana de seguimiento 5,3 meses. 
Muchos factores extrínsecos pueden producir la alteración de los mecanismos normales de defensa. Entre estos podemos mencionar la dieta y las drogas que modulan la flora intestinal, como pre y probióticos, supresores del ácido gástrico como inhibidores de la bomba de protones, bloqueadores H2, antibióticos y fármacos que alteran la motilidad ${ }^{8}$.

Se sabe que existe una dificultad en la identificación histológica de los tumores periampulares (adenocarcinoma duodenal, adenocarcinoma periampular, adenocarcinoma de cabeza de páncreas y colangiocarcinoma distal), y es posible tener un error diagnóstico al final del análisis. Con la intención de corregir este problema, enviamos todas las biopsias a una revisión retrospectiva por un médico anatomopatólogo. Estudios recientes mencionan la importancia del estudio sistematizado de las piezas quirúrgicas, y el uso de un panel inmunohistoquímico para la precisión diagnóstica ${ }^{11,12}$. Es de mayor importancia la subclasificación histológica de estos pacientes, más que categorizarlos desde un punto de vista anatómico. De esta manera una separación en una histología de predominio intestinal versus biliopancreático, tiene una implicancia pronóstica y terapéutica. Los pacientes del primer grupo presentan mejor pronóstico que los del segundo, independientemente de su ubicación, y son beneficiados de quimioterapia basados en el 5-fluoracilo, a diferencia del segundo grupo que se beneficia de esquemas basados en gemcitabina. Marcadores como el CDX2 (tipo intestinal) y el MUC1 (tipo biliopancreático) son necesarios ${ }^{13,14}$.

La diferencia etaria y de sobrevida entre las lesiones poliposas y carcinomas permite sugerir una correlación entre la edad del paciente y el riesgo de malignización del pólipo adenomatoso, al igual que el mecanismo del cáncer de colon. Este hallazgo nos permite proponer una endoscopía digestiva alta de tamizaje en ciertas poblaciones de riesgo. Este examen no solo permite observar el duodeno, sino que también lesiones esofágicas y gástricas, estas últimas muy frecuentes en nuestro medio ${ }^{15}$.

El hecho de contar con un alto porcentaje de pacientes con pólipos adenomatosos ubicados en la zona de la papila duodenal, nos hace correlacionar estas lesiones con eventuales casos de poliposis familiar múltiple, por lo que se plantea la posibilidad de posteriores estudios correlacionando eventuales mutaciones y hallazgos a la colonoscopía de estos pacientes ${ }^{16}$.

La gran mayoría de los pacientes con adenocarcinoma duodenal presentaron síntomas al momento del diagnóstico, el más frecuente fue la ictericia. En cuanto a los pacientes con pólipos la presencia de sintomatología al momento del diagnóstico es menor, y el síntoma más frecuente fue el dolor abdominal. Lo anterior nos permite concluir que en lesiones benignas la sintomatología es más vaga y en malignas es más específica de la zona. Lo descrito se correlaciona con los hallazgos en la literatura ${ }^{17,18}$.

En cuanto a los métodos de estudio el hecho que la gran mayoría de los pacientes en sus etapas avanzadas contase con un TC, una endoscopía digestiva alta, y un número importante con una CPRE, nos hace proponer que estos pacientes debiesen ser estudiados y tratados en centros de alta complejidad, con equipos multidisciplinarios, que tengan las competencias para resolver esta patología.

La menor sobrevida global entre pacientes con adenopatías positivas, T4 y presencia de metástasis a distancia, concuerda con la literatura existente ${ }^{2,11}$.

El hecho que los pacientes sometidos a DPC, y aún más los pacientes tratados con quimioterapia, y quimioterapia asociado a radioterapia, presentaron una sobrevida global mayor, nos hace proponer que todo paciente con enfermedad localizada debiese ser tratado con intención curativa. Se desconoce el posible efecto de una neoadyuvancia en este grupo de pacientes y su efecto en la calidad de vida, se abre la puerta a posibles estudios posteriores ${ }^{19,20}$.

Un déficit de nuestro estudio es el número de pacientes, el cual no permite realizar conclusiones con poder estadístico en algunos subgrupos. Existe una incidencia elevada de adenocarcinomas duodenales en nuestra población en comparación a otros estudios ${ }^{3,17}$, lo cual hace suponer posibles mecanismos subyacentes a ser estudiados. Debido a la particular relación del duodeno y los órganos vecinos, en especial la segunda porción duodenal, los factores de riesgo no solo podrían estar en relación con características propias de este segmento, sino que también de factores provenientes del páncreas, árbol biliar y cámara gástrica. Estos 2 últimos con neoplasias de alta frecuencia en nuestro medio. Son necesarios futuros estudios respecto a la presencia de Helicobacter pylori, litiasis biliar y su composición, y posibles factores genéticos asociados ${ }^{16,21-23}$.

\section{Responsabilidades éticas}

Protección de personas y animales. Los autores declaran que para esta investigación no se han realizado experimentos en seres humanos ni en animales.

Confidencialidad de los datos. Los autores declaran que en este artículo no aparecen datos de pacientes.

Conflictos de interés: no hay. 


\section{Agradecimientos}

Ilse López B, Ricardo Cerda S, Rafael Moya
A, Francisco Podestá H, Nicole Gutiérrez P, Erik Manríquez A, Susana Fuenzalida Z, Federico Hernández $\mathrm{F}$.

\section{Bibliografía}

1. Chen N, Zhang TL. Clinicals characteristics and diagnosis and treatment of malignant tumor of the duodenum. Journal of Peking University (Health Science) 2012;44:942-5.

2. Liang TJ, Wang BW, Liu SI, Chou NH, Tsai CC, Chen IS, et al. Number of involved lymph nodes is important in the prediction of prognosis for primary duodenal adenocarcinoma. Journal of Chinese medical association 2012;75:57380.

3. Lepage C, Bouvier AM, Manfredi S, Dancourt V, Faivre J. Incidence and Management of Primary Malignant Small Bowel Cancers: A Well-defined French Population Study. Am J Gastroenterol. 2006;101:2826-32.

4. Cunningham JD, Aleali R, Aleali M, Brower ST, Aufses AH. Malignant small bowel neoplasms. Histopatologic determinants of recurrence and survival. Ann Surg. 1997;225:300-6.

5. Bilimoria KY, Bentrem DJ, Wayne JD, Ko CY, Bennett L, Talamonti MS. Small Bowel Cancer in the United States Changes in Epidemiology, Treatment, and Survival Over the Last 20 Years. Ann Surg. 2009;249:63-71.

6. Hatzaras I, Palesty JA, Abir F, Sullivan P, Kozol R, Dudrick SJ, et al. Small bowel tumors. Epidemiologic and clinical characteristics of 1260 cases from the Connecticut tumor registry. Arch Surg. 2007;142:229-35.

7. Ovesen L, Bendtsen F, Tage-Jensen U, Pedersen NT, Gram BR, Rune SJ. Intraluminal $\mathrm{pH}$ in the stomach, duodenum, and proximal jejunum in normal subjects and patients with exocrine pancreatic insufficiency Gastroenterology 1986;90:958-62.

8. Ghoshal UC, Shukla R, Ghoshal U. Small Intestinal Bacterial Overgrowth and Irritable Bowel Syndrome: A Bridge between Functional Organic Dichotomy. Gut and Liver 2017;11:196-208.

9. Mima K, Ogino S, Nakagawa S, Sawayama H, Kinoshita K, Krashima $\mathrm{R}$, et al. The role of intestinal bacteria in the development and progression of gastrointestinal tract neoplasms. Surg Oncol. 2017;26:368-76.

10. Holmes G, Idunn G, Cockel R, Brookes $\mathrm{S}$. Adenocarcinoma of the upper small bowel complicating coeliac disease. Gut 1980;21:1010-16.

11. Elebro J, Jirström K. Use of a standardized diagnostic approach improves the prognostic information of histopathologic factors in pancreatic and periampullary adenocarcinoma. Diagnostic Pathology 2014;9:80.

12. Fernández $\mathrm{C}$, Fernández-Woodbridge $\mathrm{A}$, Alistair M, Zhang Q, Bozoky B, Vasan $\mathrm{S}$, et al. Immunohistochemical Typing of Adenocarcinomas of the Pancreatobiliary System Improves Diagnosis and Prognostic Stratification. Plos One 2016;11:1-20.

13. Ushiku T, Arnason T, Fukuyama M, Lauwers G. Extra-ampullary adenocarcinoma. Am J Surg Pathol. 2014;38:1484-93.

14. Williams J, Chan C, Toste P, Elliot I, Vasquez C, Sunjaya D, et al. Association of histopathologic phenotype of periampullary adenocarcinomas with survival. JAMA Surg. 2017;152:82-8.

15. Rollán A, Cortés P, Calvo A, Araya R, Bufadel ME, González R, et al. Diagnóstico precoz de cáncer gástrico.
Propuesta de detección y seguimiento de lesiones premalignas gástricas: protocolo ACHED. Rev Med Chile 2014;142:118192.

16. Bjork $\mathrm{J}$, Akerbrant $\mathrm{H}$, Iselius $\mathrm{L}$, Bergman A, Engwall Y, Wahlstrom J, Periampullary Adenomas and Adenocarcinomas in Familial Adenomatous Polyposis: Cumulative Risks and $A P C$ Gene Mutations. Gastroenterology 2001;121:1127-35.

17. Solej M, D'Amico S, Brondino G, Ferronato M, Nano M. Primary duodenal adenocarcinoma. Tumori 2008;94:779-86.

18. Catán F, Núñez J, Astudillo C, Uribe M, Uribe-Echeverría S. Ictericia postoperatoria. Gastroenterol Latinoam. 2014;25:54-7.

19. Arancibia H, Carvajal C, Bustamante J, Talhouk O, Guler C, et al. Análisis de calidad de vida en pacientes gastrectomizados por cáncer gástrico. Rev Med Chile 2009;137:481-6.

20. Morales A, Cavada G, Miranda J, Ahumada M, Leo D. Eficacia del programa alivio del dolor por cáncer avanzado y cuidados paliativos de Chile. Revista del Dolor 2013;59:18-25.

21. Segura-López FK, Güitrón-Cantú A, Torres J. Association between Helicobacter spp. infections and hepatobiliary malignancies: A review. World J Gastroenterol. 2015;21:1414-23

22. Iyer P, Barreto SG, Sahoo B, Chandrani P, Ramadwar MR, Shrikhande S, et al. Non-typhoidal Salmonella DNA traces in gallbladder cancer. Infect Agent Cancer 2016;11:12. Published online 2016 Mar 3. doi: 10.1186/s13027-016-0057-x

23. Navarro D, Durán S. Gallbladder cancer and nutritional risk factors in Chile. Nutr Hosp. 2016;33:105-10. 